\title{
MicroRNA-29a/b/c targets iNOS and is involved in protective remote ischemic preconditioning in an ischemia-reperfusion rat model of non-alcoholic fatty liver disease
}

\author{
YUN-FEI DUAN, DONG-LIN SUN, JING CHEN, FENG ZHU and YONG AN \\ Department of Hepatobiliary Surgery, The Third Affiliated Hospital of Soochow University, \\ Changzhou, Jiangsu 213000, P.R. China
}

Received June 21, 2015; Accepted October 24, 2016

DOI: $10.3892 / 01.2017 .5623$

\begin{abstract}
Remote ischemic preconditioning (RIPC) protects against the injury that is incurred by ischemia and reperfusion (IR); however, the role of RIPC in liver IR injury in non-alcoholic fatty liver disease (NAFLD) remains unclear. In this study, a NAFLD rat model was utilized in a series of different surgical procedures and molecular experiments. Rats of the IR group and the RIPC+IR group exhibited more severe injury than NAFLD control rats (in which the liver was prodded following a median-incision laparotomy). The liver condition, measured by serum alanine transaminase and aspartate transaminase levels, of the RIPC+IR group was better than that of the IR group. In addition, alanine transaminase and aspartate transaminase levels were lower in the RIPC+IR group compared with the IR group $(\mathrm{P}<0.001)$. Flow cytometry revealed that the cell apoptosis ratio was significantly lower in the RIPC+IR group than in the IR group $(\mathrm{P}<0.001)$. Reverse transcription-polymerase chain reaction (RT-qPCR) was used to assess miR-29a/b/c levels, revealing that they were significantly reduced in the RIPC and RIPC+IR groups, but did not vary in the IR group compared with the control group. RT-qPCR also revealed that iNOS mRNA levels were not significantly different among any of the NAFLD groups; however, western blot analysis indicated that iNOS protein levels were increased in the RIPC group and the RIPC+IR group compared with the control and IR groups. A luciferase reporter assay demonstrated that transfection with miR-29a/b/c mimics significantly decreased the luciferase activities of plasmids containing the wild-type iNOS 3'-untranslated region (UTR) (relative fluorescence intensity: $0.47 \pm 0.06$ for miR-29a, $0.36 \pm 0.07$ for miR-29b, $0.41 \pm 0.04$ for miR-29c; $\mathrm{P}<0.001$ ), whereas the activities of plasmids containing the mutant iNOS
\end{abstract}

Correspondence to: Dr Yong An, Department of Hepatobiliary Surgery, The Third Affiliated Hospital of Soochow University, 185 Juqian Street, Changzhou, Jiangsu 213000, P.R. China E-mail: anyong8080@163.com

Key words: remote ischemic preconditioning, microRNA-29a/b/c, inducible nitric oxide synthase, non-alcoholic fatty liver disease
3'-UTR sequence were not markedly affected [relative fluorescence intensity: $0.99 \pm 0.08$ for miR-29a $(P=0.1349), 0.99 \pm 0.09$ for miR-29b $(\mathrm{P}=0.1607), 0.97 \pm 0.07$ for miR-29c $(\mathrm{P}=0.1824)]$. This suggested that miR-29a/b/c downregulates iNOS by directly targeting its 3'-UTR. In summary, the results suggest that RIPC has a protective effect in NAFLD liver IR injury, which may be due to reduced $\mathrm{miR}-29 \mathrm{a} / \mathrm{b} / \mathrm{c}$ levels in the skeletal muscle, leading to increased iNOS and, therefore, nitric oxide.

\section{Introduction}

In recent years, the incidence of non-alcoholic fatty liver disease (NAFLD) has increased (1). Patients with fatty liver degeneration have twice the risk of complications following liver resection compared with patients without liver disease; these complications may include infective, wound-related (seroma, infection and hernia), hepatobiliary (cholangitis, biliary obstruction, liver failure, hepatic artery infusion pump failure, ascites, perihepatic fluid collection and perihepatic abscess) and gastrointestinal complications (gastrointestinal hemorrhage, bowel obstruction, paralytic ileus, infectious diarrhea, fistula, pancreatitis and esophagitis). Additionally, the risk of mortality for patients with severe steatosis following liver resection is almost three-times greater than that for patients without liver steatosis (2). Compared to a normal liver, a liver with fatty degeneration more easily suffers from ischemia reperfusion (IR) injury. Treatment with omega-3 fatty acids significantly reduces hepatic steatosis, resulting in significantly reduced IR injury (3). Proanthocyanidins from grape seeds have a protective effect against liver IR injury, particularly in mice with diet-induced obesity (4). However, the use of drugs to treat fatty liver degeneration is an indirect strategy for reducing injury; the treatment can enhance liver function and thus increase the tolerance of the liver to IR damage, but not reduce IR injury directly.

Increasing evidence indicates that remote ischemic preconditioning (RIPC) represents a strategy for harnessing the body's endogenous protective capabilities to counter the injury that is incurred by IR in various organs (5-13), including the normal liver (14-17). The protective mechanism of RIPC is highly complex (18-22) and involves the improvement of hepatic oxygenation (23), reduction of serum cytokine-induced 
neutrophil chemoattractant-1 levels (24), modulation of hepatic microcirculation (15) and activation of the soluble guanylate cyclase-cyclic guanosine monophosphate pathway (20), resulting in protective effects against IR injury. Notably, Abu-Amara et al (25) suggested that nitric oxide (NO) is an essential mediator of the protection that is afforded by hind-limb RIPC against liver IR injury. The mechanisms underlying this protective effect involve the preservation of the sinusoidal structure and the maintenance of blood flow through the hepatic microcirculation (25). However, the mechanism by which RIPC increases NO, and the role and mechanism of RIPC in NAFLD liver IR injury remain unclear.

Therefore, in the present study, a NAFLD rat model was utilized in a series of different surgical procedures and molecular experiments. The data indicate that RIPC has a protective effect on NAFLD liver IR injury. RIPC may exert this effect by reducing expression levels of the microRNAs (miRNAs) miR-29a/b/c in the skeletal muscle, subsequently increasing inducible NO synthase (iNOS) and thus increasing NO. miR-29a/b/c targets iNOS, which plays an important role in the protective effect of RIPC in NAFLD liver IR injury.

\section{Materials and methods}

Cell cultures and tissue collections. The skeletal muscle cell line $\mathrm{C} 2 \mathrm{C} 12$ was purchased from the Shanghai Cell Bank (Shanghai, China) and cultured in Dulbecco's modified Eagle's medium (DMEM) (Invitrogen; Thermo Fisher Scientific, Inc., Carlsbad, CA, USA) that was supplemented with $10 \%$ fetal bovine serum (FBS) (Sigma-Aldrich; Merck Millipore, Bedford, MA, USA). The rats were purchased from The Model Animal Research Center of Nanjing University (Nanjing, China). The animal studies were approved by the Ethics Committee of Soochow University.

Establishment of the animal models. To establish the NAFLD rat model, specific pathogen-free-grade Sprague Dawley male rats weighing $200 \mathrm{~g}$ were fed a high-fat diet containing $2 \%$ cholesterol, $0.5 \%$ sodium cholate, $0.2 \%$ propylthiouracil, $5 \%$ sugar, $10 \%$ lard, and $82.3 \%$ basic feed. The rats were maintained in a temperature-controlled environment with $40-70 \%$ humidity and fed for 5 weeks.

To establish the NAFLD/liver IR rat model, NAFLD rats were anesthetized with $10 \%$ chloral hydrate by intraperitoneal injection $(350 \mathrm{mg} / \mathrm{kg}$ ). Laparotomy was subsequently performed with a median incision. The perihepatic ligament was separated, and the blood supply to the hepatic left lateral lobe, left interior lobe and middle lobe was blocked using a metal microvascular clamp, resulting in $70 \%$ liver ischemia.

To establish the NAFLD/RIPC rat model, the right hind limb of an NAFLD rat was tied up with a tourniquet such that the right femoral artery was pulseless for $5 \mathrm{~min}$. The tourniquet was then released to restore the blood flow for $5 \mathrm{~min}$. These two procedures were repeated 6 times.

Rats were sacrificed by spinal dislocation immediately at the end of experimental process.

Experimental groups. In the control group, the liver was prodded following a median-incision laparotomy. In the RIPC group, the hind limb was ischemic for $5 \mathrm{~min}$, followed by reperfusion for $5 \mathrm{~min}$. After 6 cycles, the hind limb underwent reperfusion for $160 \mathrm{~min}$. In the IR group, the blood supply to the hepatic left lateral lobe, left interior lobe and middle lobe was blocked for $40 \mathrm{~min}$, followed by reperfusion for $120 \mathrm{~min}$. In the RIPC+IR group, following 6 cycles of hind limb ischemia and reperfusion, liver ischemia was performed for $40 \mathrm{~min}$, and reperfusion was given for $120 \mathrm{~min}$. A total of 6 rats were allocated to each group in the study.

Pathological examination. The left lobe of the liver was resected and frozen in liquid nitrogen following the surgical procedure. For the pathological examination, 4- $\mu$ m-thick tissue sections were fixed, rinsed, stained with hematoxylin and eosin (HE), dehydrated through increasing concentrations of ethanol and xylene, and blocked by neutral gum. Pathological observation was performed by two pathologists.

Liver function test. Hepatic venous blood was drawn from the rats following the surgical procedure. The serum alanine transaminase (ALT) and aspartate transaminase (AST) levels were measured by biochemical analysis using ALT and AST assay kits (Beckman Coulter, Inc., Brea, CA, USA) with a Beckman Coulter Chemistry Analyzer AU5800 series (Beckman Coulter K.K., Tokyo, Japan).

Cell apoptosis analysis. Fresh liver tissue was minced with scissors and digested with $0.02 \%$ protease and $0.05 \%$ collagenase $\mathrm{P}$ (Roche, Basel, Switzerland) in a balanced salt solution for $30 \mathrm{~min}$ at $37^{\circ} \mathrm{C}$. The suspension containing the cells and tissues was filtered through a 200-nm nylon mesh. The cell pellets were collected, washed with PBS, suspended in $100 \mu \mathrm{l}$ of $1 \mathrm{X}$ binding buffer, and stained with $5 \mu \mathrm{l}$ of fluorescein isothiocyanate (FITC)-Annexin V and a 1- $\mu$ l working solution of propidium iodide (PI) $(100 \mu \mathrm{g} / \mathrm{ml})$ at room temperature for $15 \mathrm{~min}$ in the dark. The stained cells were immediately analyzed by flow cytometry.

Reverse transcription-quantitative polymerase chain reaction (RT-qPCR). Total RNA was extracted from skeletal muscle and cell lines using TRIzol reagent (Invitrogen; Thermo Fisher Scientific, Inc.). For tissue RNA extraction, $100 \mathrm{mg}$ of frozen muscle in liquid nitrogen was ground and suspended in $1 \mathrm{ml}$ of TRIzol. For total RNA extraction from cells, $1 \times 10^{7}$ cell pellets were collected, washed and resuspended in $1 \mathrm{ml}$ of TRIzol.

To analyze mRNA, cDNA was synthesized using the PrimeScript RT Kit (Takara, Dalian, China). qPCR was performed with FastStart Universal SYBR Green Master Mix (ROX) (Roche Diagnostics, Indianapolis, IN, USA) with an ABI 7500 Real-Time PCR System (Applied Biosystems; Thermo Fisher Scientific, Inc.). The reaction mixture contained 12.5 $\mu 1$ FastStart Universal SYBR Green Master Mix (ROX), $0.5 \mu \mathrm{l}$ forward primer $(30 \mu \mathrm{M}), 0.5 \mu \mathrm{l}$ reverse primer $(30 \mu \mathrm{M})$, $9.5 \mu \mathrm{l}$ PCR-grade water and $2.5 \mu \mathrm{l}$ of template cDNA (25 ng). Thermal cycling conditions for qPCR were $50^{\circ} \mathrm{C}$ for $2 \mathrm{~min}$ and $95^{\circ} \mathrm{C}$ for $10 \mathrm{~min}$, followed by $95^{\circ} \mathrm{C}$ for $15 \mathrm{sec}$ and $58^{\circ} \mathrm{C}$ for $30 \mathrm{sec}$ for 40 cycles. Each reaction was analyzed in triplicate. Relative mRNA expression was examined as the inverse log of the $\Delta \Delta \mathrm{Cq}$ and normalized to the expression of the reference gene GAPDH (26). The primers for qPCR were synthesized by Invitrogen (Thermo Fisher Scientific, Inc., Shanghai, China), 
and the sequences were as follows: iNOS sense, 5'-AGGACG AGAAGCGGAGACC-3'; iNOS antisense, 5'-CATGAGCAA AGGCGCAGAA-3'; GAPDH sense, 5'-TCACCCACACTG TGCCCATCTACGA-3'; and GAPDH antisense, 5'-CAGCGG AACCGCTCATTGCCAATGG-3'.

For miRNA analysis, samples of total RNA (500 ng each) were reverse-transcribed into cDNA with $\mathrm{miR}-29 \mathrm{a} / \mathrm{b} / \mathrm{c}$ reverse transcriptase primers using TaqMan MicroRNA Reverse Transcription Kits [\#4366596 (miR-29a/002112, miR-29b/000413, miR-29c/000587); Applied Biosystems; Thermo Fisher Scientific, Inc.]. The RT master mix included the following: $5 \mu \mathrm{l}$ RNA samples (10 ng), $0.15 \mu 1$ dNTPs, $1 \mu 1$ reverse transcriptase, $1.5 \mu 1$ RT buffer, $0.19 \mu 1$ RNase inhibitor, $3.16 \mu 1$ nuclease-free water and $4 \mu \mathrm{l}$ of RT primers for miR-29a/b/c and U6 (1 $\mu \mathrm{l}$ each) up to a total volume of $15 \mu \mathrm{l}$. The final mixture was centrifuged and incubated on ice for 5 minutes. The thermal program for RT was $30 \mathrm{~min}$ at $16^{\circ} \mathrm{C}, 30 \mathrm{~min}$ at $42^{\circ} \mathrm{C}, 5 \mathrm{~min}$ at $85^{\circ} \mathrm{C}$ and hold at $4^{\circ} \mathrm{C}$. The levels of $\mathrm{miR}-29 \mathrm{a} / \mathrm{b} / \mathrm{c}$ and U6 expression were determined by qPCR with TaqMan MicroRNA Assays [\#4427975 (miR-29a/002112, miR-29b/000413, miR-29c/000587); Applied Biosystems; Thermo Fisher Scientific, Inc.]. The qPCR reaction mixture contained $1 \mu \mathrm{l}$ TaqMan MicroRNA Assays (20X; including primers), $2 \mu \mathrm{l}$ product from RT reaction, $10 \mu 1$ TaqMan Universal PCR Master Mix II $(2 \mathrm{X})$ and $7 \mu \mathrm{l}$ nuclease-free water up to a total volume of $20 \mu \mathrm{l}$. The final mix was subjected to the following program of heating using an ABI 7500 Real-Time PCR System: 2 min at $50^{\circ} \mathrm{C} ; 10 \mathrm{~min}$ at $95^{\circ} \mathrm{C}$; then $15 \mathrm{sec}$ at $95^{\circ} \mathrm{C}$ and $60 \mathrm{sec}$ at $60^{\circ} \mathrm{C}$ for 40 cycles. The levels of mature $\mathrm{miR}-29 \mathrm{a} / \mathrm{b} / \mathrm{c}$ expression were then normalized to U6 and calculated as the inverse log of the $\Delta \Delta \mathrm{Cq}$.

Western blotting. Skeletal muscle tissue $(100 \mathrm{mg})$ or cells $\left(1 \times 10^{7}\right)$ were lysed using radioimmunoprecipitation assay buffer with $1 \%$ phenylmethane sulfonyl fluoride on ice. The total protein concentration was determined using a bicinchoninic acid assay kit (Keygen, Nanjing, China). Equal amounts of protein $(30 \mu \mathrm{g})$ were resolved by $10 \%$ SDS-PAGE and transferred to polyvinylidene difluoride membranes (EMD Millipore, Billerica, MA, USA) using a Mini Trans-Blot apparatus (Bio-Rad Laboratories, Inc., Hercules, CA, USA). The membranes were blocked in Tris-buffered saline with Tween-20 (TBST) containing 5\% non-fat milk and probed with rabbit anti-rat monoclonal iNOS primary antibodies (1:10; \#ab15323; Abcam, Cambridge, MA, USA) overnight at $4^{\circ} \mathrm{C}$. An anti-GAPDH antibody (1:1,000; AG1019; Beyotime, Nantong, China) was used as an internal control. Subsequently, the membranes were washed with $3 \mathrm{X}$ TBST prior to incubation with horseradish peroxidase-conjugated goat anti-rabbit secondary antibodies (1:1,000; \#A0192; Beyotime, Nantong, China) for $2 \mathrm{~h}$ at room temperature. The membranes were then washed with $3 \mathrm{X}$ TBST and $1 \mathrm{X}$ TBS. For image development, Immobilon Western Chemiluminescent HRP Substrate (\#WBKLS0500; EMD Millipore) was used. Bands on the membrane were developed using a ChemiImager 5500 Imaging System (Alpha Innotech Co., San Leandro, CA, USA).

miRNA transfection. miRNA transfection was performed using Lipofectamine 2000 (Invitrogen; Thermo Fisher Scientific, Inc., Carlsbad, CA, USA). The miR-29a/b/c mimic, inhibitor and control were designed and synthesized by GenePharma, Co., Ltd. (Shanghai, China). The sequences were as follows: miR-29a-mimic, 5'- UAGCACCAUCUGAAAUCG GUUA-3'; miR-29b-mimic, 5'-UAGCACCAUUUGAAAUCA GUGUU-3'; miR-29c-mimic, 5'- UAGCACCAUUUGAAA UCGGUUA-3'; mimic control, 5'- UUCUCCGAACGUGUC ACGUTT-3'; miR-29a inhibitor, 5'-UAACCGAUUUCAGAU GGUGCUA-3'; miR-29b inhibitor, 5'-AACACUGAUUUC AAAUGGUGCUA-3'; miR-29c inhibitor, 5'-UAACCGAUU UCAAAUGGUGCUA-3'; inhibitor control, 5'-CAGUACUUU UGUGUAGUACAA-3'.

For transfection, $\mathrm{C} 2 \mathrm{C} 12$ skeletal muscle cells were plated at $1.0 \times 10^{6}$ cells/well in 6 -well plates. Lipofectamine 2000 (5 $\mu \mathrm{l}$ ) was added into $250 \mu \mathrm{l}$ DMEM at room temperature and stood for $5 \mathrm{~min}$. In addition, miRNA-mimics or inhibitors for miR-29a, $-29 b$ or $-29 \mathrm{c}$, respectively $(5 \mu \mathrm{l})$, were added into $250 \mu \mathrm{l}$ DMEM at room temperature and stood for $5 \mathrm{~min}$. Subsequently, the two solutions were mixed and allowed to incubate for $20 \mathrm{~min}$. Prior to transfection, cell culture medium was removed and the cells were incubated in $2 \mathrm{ml} \mathrm{DMEM}$. The aforementioned 510- $\mu 1$ mixture was added into the corresponding wells. The transfected cells were incubated in a humidified chamber at $37^{\circ} \mathrm{C}$ with $5 \% \mathrm{CO}_{2}$ for $24 \mathrm{~h}$. Total RNA and protein were extracted at $24 \mathrm{~h}$ post-transfection and used for RT-qPCR and western blot analyses.

Luciferase reporter assay. Luciferase reporter constructs were generated by ligating 60-bp synthetic oligonucleotides (Invitrogen; Thermo Fisher Scientific, Inc., Shanghai, China) containing putative miRNA binding sites (or their mutant versions) from the 3'-untranslated region (3'-UTR) of iNOS to the XbaI-FseI sites of the pGL3-control vector (Promega Corporation, Madison, WI, USA). Successful cloning was verified by sequencing. At $24 \mathrm{~h}$ prior to transfection, $\mathrm{C} 2 \mathrm{C} 12$ skeletal muscle cells were plated at $1.5 \times 10^{5}$ cells/well in 24 -well plates, and $200 \mathrm{ng}$ of each independent luciferase reporter plasmid plus $80 \mathrm{ng}$ of pRL-TK (Promega Corporation) were transfected, together with $60 \mathrm{pmol}$ of miR-29a/b/c mimics, inhibitors or control miRNAs using Lipofectamine 2000. The luciferase activity was measured at $48 \mathrm{~h}$ following transfection using the Dual-Luciferase Reporter Assay System (Promega Corporation). Firefly luciferase activity was normalized to Renilla luciferase activity in each transfected well.

Statistical analysis. All experiments were repeated in triplicate and the values presented are the mean \pm standard deviation. Statistical significance was determined with Student's $t$-test using SPSS 15.0 software (SPSS, Inc., Chicago, IL, USA) $\mathrm{P}<0.05$ was considered to indicate a statistically significant difference.

\section{Results}

Establishment of the NAFLD rat model. As shown in Fig. 1, in contrast to the livers of the rats that were fed a normal diet, the livers of the rats that were fed a high-fat diet for five weeks were pale yellow, soft and smooth on the surface; however, no focal nodule formation was observed (Fig. 1A and B). HE staining revealed that the rats that were fed the high-fat diet developed hepatic steatosis, particularly around the liver lobes. These liver 

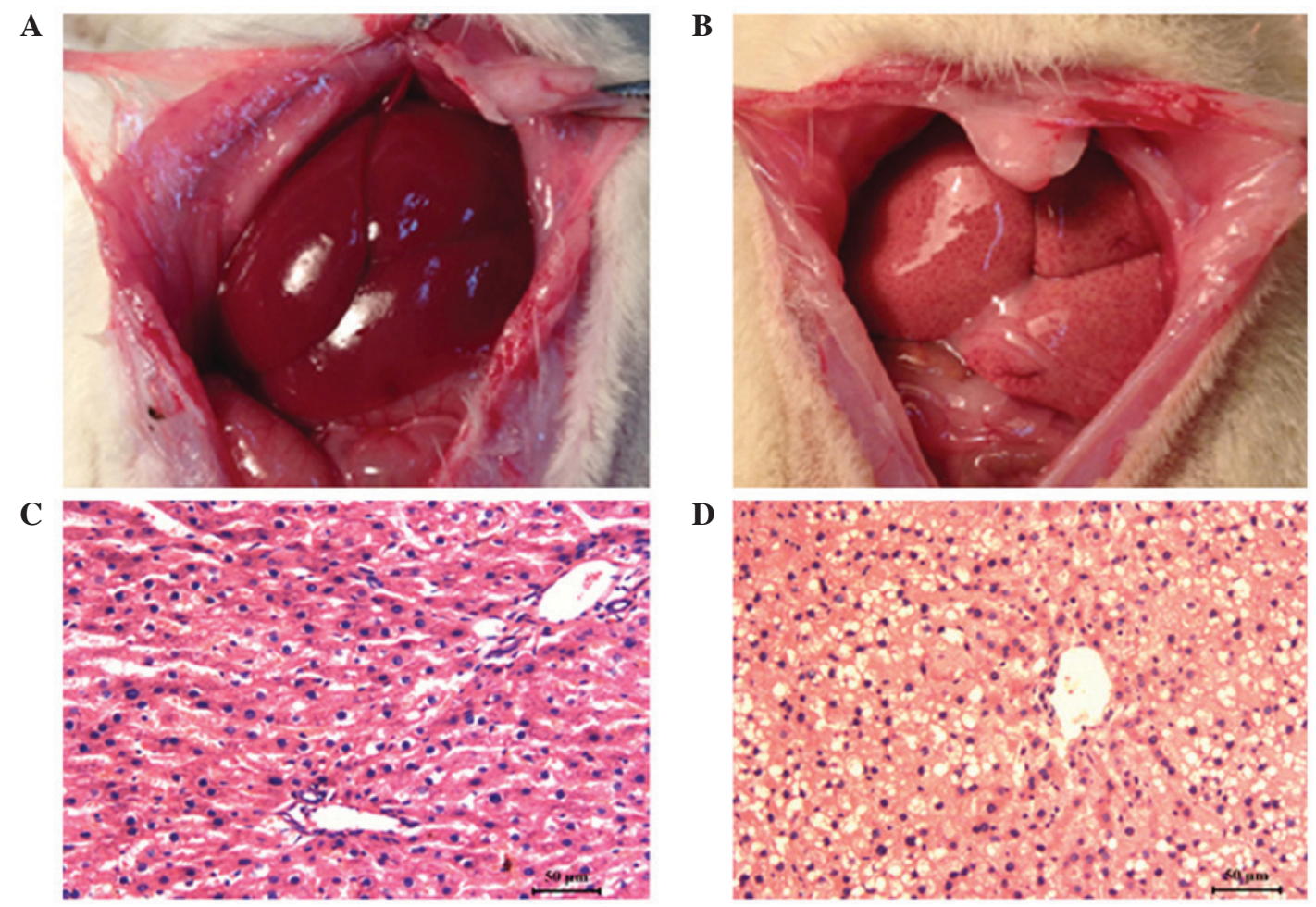

Figure 1. Establishment of the NAFLD rat model. (A) Gross appearance of normal liver; (B) gross appearance of liver of an NAFLD rat; (C) HE staining of a normal liver; (D) HE staining of an NAFLD rat liver. Scale bar, $50 \mu \mathrm{m}$. NAFLD, non-alcoholic fatty liver disease; HE, hematoxylin and eosin.

cells were swollen and round, with large vacuoles in the cytoplasm. These morphological changes indicated that the NAFLD rat model had been successfully established (Fig. 1C and D).

RIPC has a protective effect on liver IR. The gross and microscopic appearances of the livers of rats following different surgical treatments are shown in Fig. 2A and B. The gross appearance indicated that the injuries incurred in rats of the IR group were more severe than those incurred in rats of the RIPC+IR group. There was no obvious difference between the RIPC group and the control group. Upon pathological observation, the control group and the RIPC group exhibited hepatic steatosis. The IR group exhibited liver cell steatosis with a few inflammatory cells infiltrating the portal area and severe edema in the liver cells. The RIPC+IR group exhibited liver steatosis in the hepatic lobule, a small amount of liver cell necrosis in the portal area, and mild edema of the liver cells. Based on the gross and microscopic appearances of the livers, rats of the IR group and the RIPC+IR group experienced more severe injuries than the rats of the control group. However, the liver condition of the RIPC+IR group was better than that of the IR group. There was no clear difference between the RIPC group and the control group.

As shown in Fig. 2C and D, the levels of ALT and AST were lower in the RIPC+IR group compared with the IR group $(\mathrm{P}<0.001)$. In addition, compared with the control group, the ALT and AST levels were significantly higher in the RIPC+IR and IR groups (both $\mathrm{P}<0.001$ ). However, there were no significant differences between the RIPC group and the control group (ALT, $\mathrm{P}=0.9246$; AST, $\mathrm{P}=0.5268$ ).

Flow cytometry revealed that the cell apoptosis ratio was significantly lower in the RIPC+IR group as compared with the
IR group ( $\mathrm{P}<0.001 ;$ Fig. 2E). The ratio of hepatocyte apoptosis was also significantly increased in the RIPC+IR group and the IR group (both $\mathrm{P}<0.001$ ) compared with the control group. No significant difference was identified between the RIPC group and the control group $(\mathrm{P}=0.4334)$. Collectively, these results indicate that RIPC has a protective effect on liver IR.

RIPC decreases the miR-29a/b/c levels in skeletal muscle and increases the iNOS protein level. $\mathrm{miR}-29 \mathrm{a} / \mathrm{b} / \mathrm{c}$ levels in skeletal muscle were examined by RT-qPCR. Compared with the control group, the miR-29a/b/c levels were found to be significantly decreased in the RIPC and RIPC+IR groups $(\mathrm{P}<0.05)$, whereas there was no variation in the IR group (miR-29a vs. control, $\mathrm{P}=0.4021$; miR-29b vs. control, $\mathrm{P}=0.3001$; miR-29c vs. control, $\mathrm{P}=0.5516$; Fig. 3A-C). No significant differences between the RIPC group and the RIPC+IR group were observed (miR-29a, $\mathrm{P}=0.6075$; miR-29b, $\mathrm{P}=0.5322$; miR-29c, $\mathrm{P}=0.4414$; Fig. $3 \mathrm{~A}-\mathrm{C})$. The iNOS mRNA levels were not significantly different in the IR $(\mathrm{P}=0.3661)$, RIPC $(\mathrm{P}=0.8991)$ or RIPC+IR ( $\mathrm{P}=0.8077)$ groups compared with the control group (Fig. 3D). However, the iNOS protein levels were increased significantly in the RIPC group and the RIPC+IR group compared with the control group, while no marked changes were observed in the IR group (Fig. 3E). There was no evident difference between the RIPC group and the RIPC+IR group. These results indicate that RIPC may decrease the level of $\mathrm{miR}-29 \mathrm{a} / \mathrm{b} / \mathrm{c}$ in skeletal muscle cells, thereby increasing the iNOS protein level, which may be the molecular mechanism by which RIPC protects against liver IR.

$m i R-29 a / b / c$ targeting of $i N O S$. iNOS was hypothesized to be a target gene of miR-29a/b/c, and the regulatory mechanism 


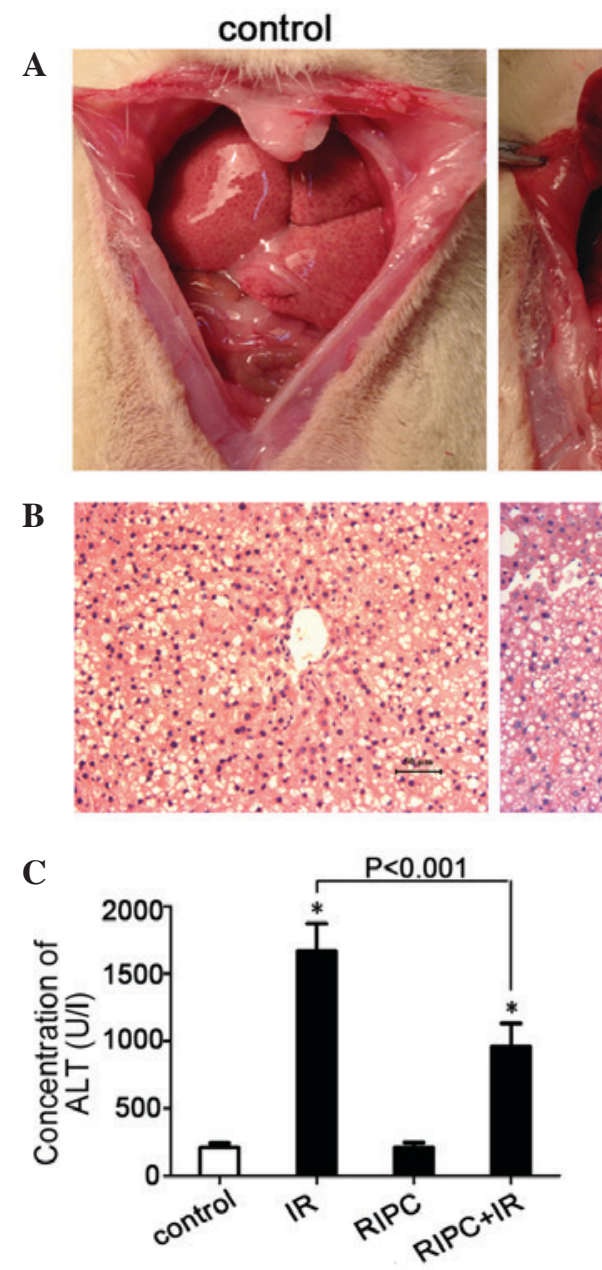

IR

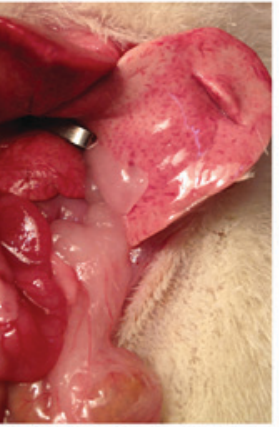

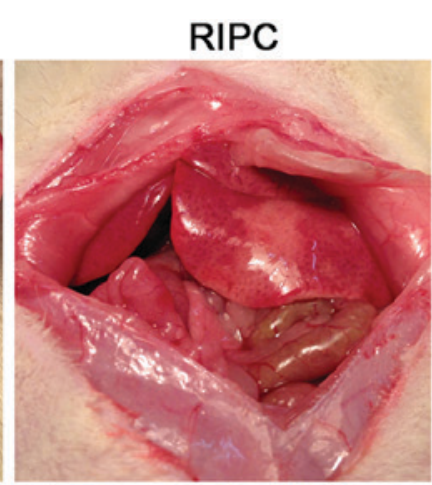

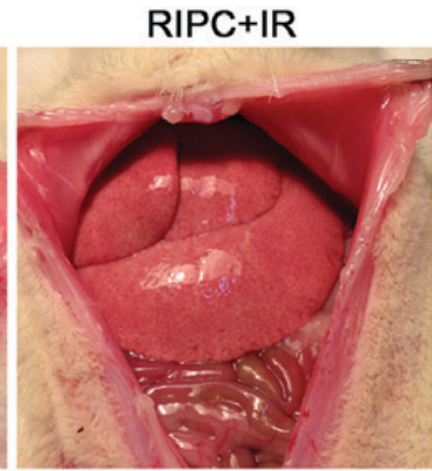

B
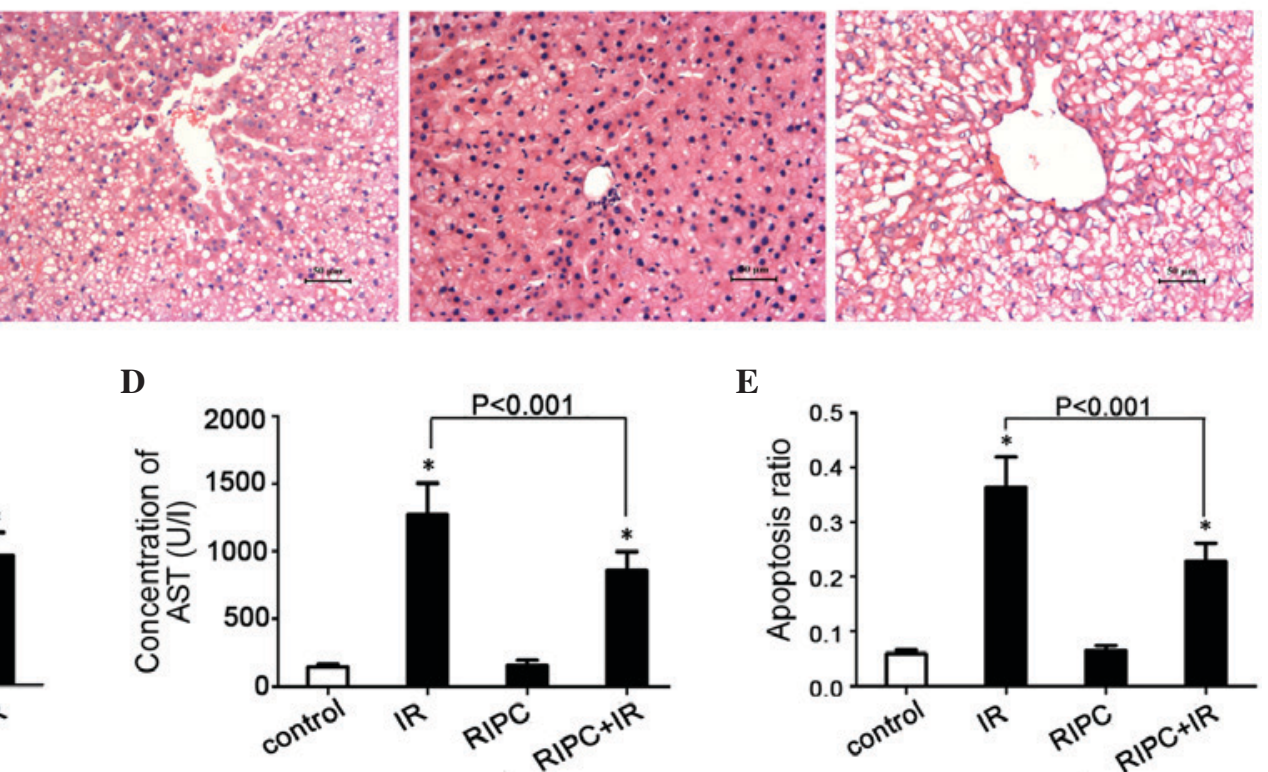

Figure 2. RIPC has a protective effect against liver IR. (A) Gross appearance of livers from rats of the control, IR, RIPC, and RIPC+IR groups. (B) Microscopic view of livers from rats of the control, IR, RIPC, RIPC+IR groups (hematoxylin and eosin staining; scale bar, $50 \mu$ m). (C) Serum concentration of ALT in each group. (D) Serum concentration of AST in each group. (E) Cell apoptosis ratio in each group. Data are presented as the mean + standard deviation ( $\mathrm{n}=6$; ${ }^{*} \mathrm{P}<0.001$ vs. control group). RIPC, remote ischemic preconditioning; IR, ischemia-reperfusion; ALT, alanine transaminase; AST, aspartate transaminase.
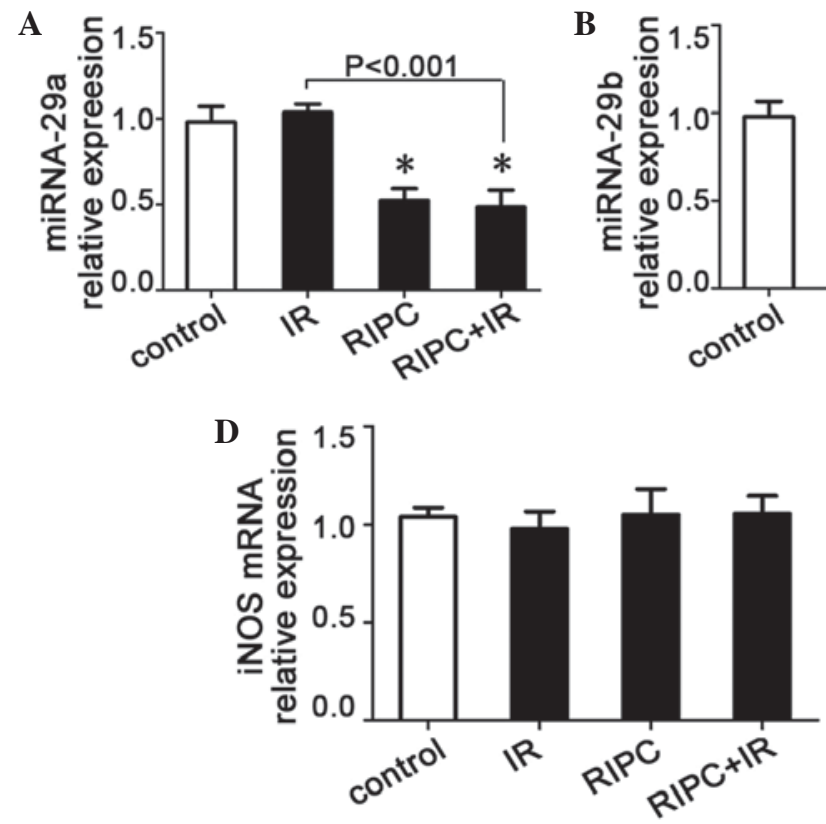
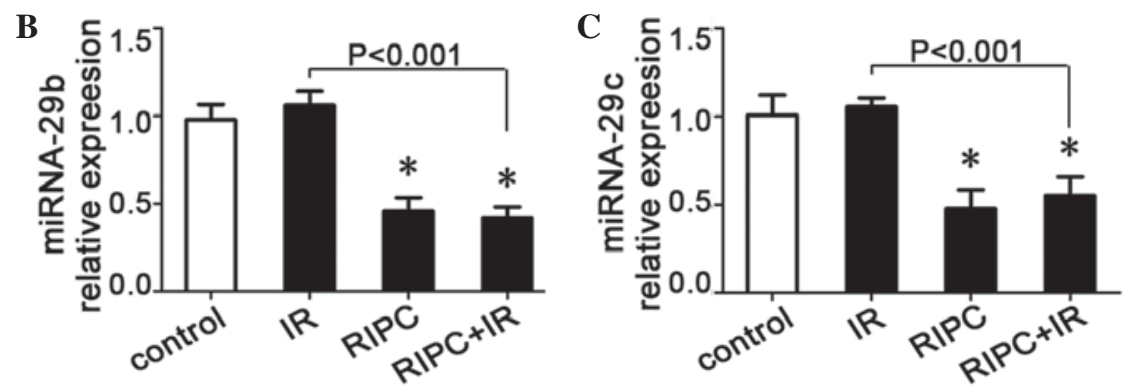

$\mathbf{E}$

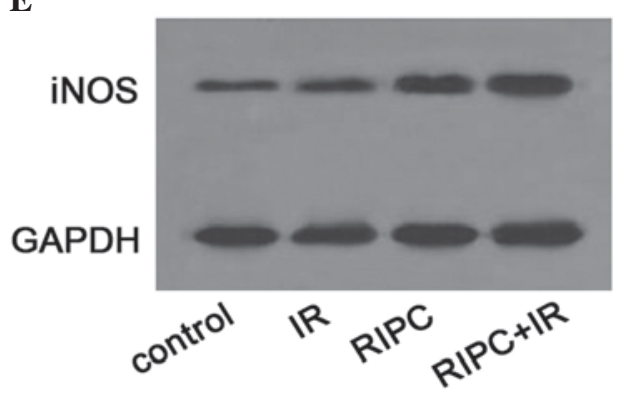

Figure 3. RIPC decreases miR-29a/b/c levels and increases iNOS protein levels. The expression levels of (A) miR-29a, (B) miR-29b and (C) miR-29c were reduced in the RIPC and RIPC+IR groups compared with the control, and in the RIPC+IR group compared with the IR group. (D) iNOS mRNA expression did not significantly vary among the groups. Data are presented as the mean + standard deviation ( $n=6 ;{ }^{*} \mathrm{P}<0.001$ vs. control group). (E) iNOS protein expression in each group. RIPC, remote ischemic preconditioning; IR, ischemia-reperfusion; miR-29, microRNA-29; iNOS, inducible nitric oxide synthase. 


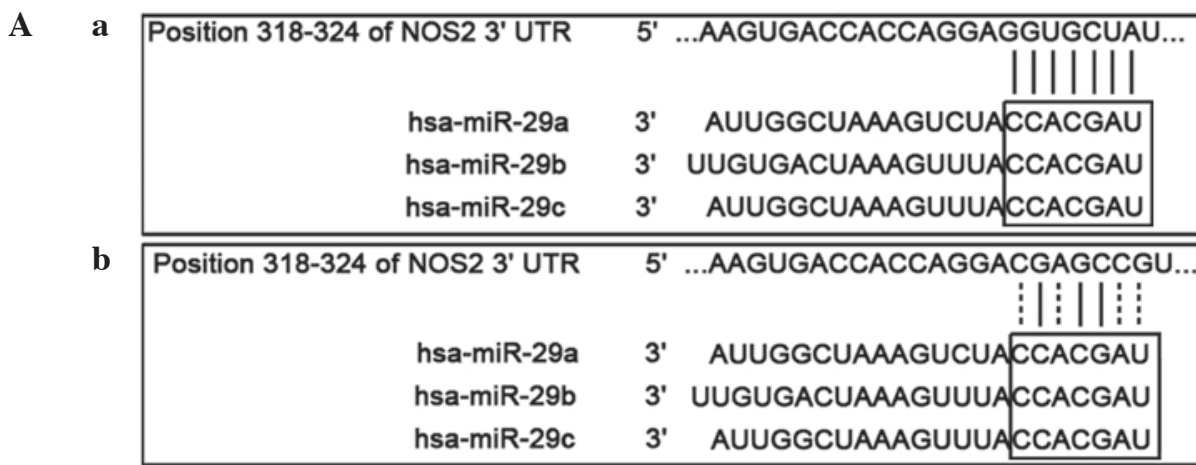

B

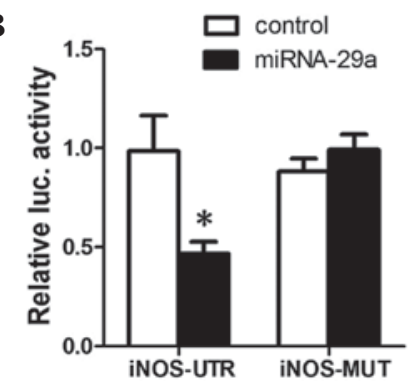

C

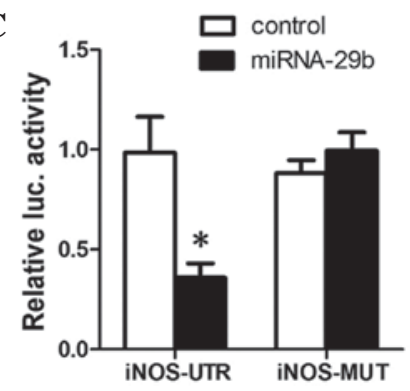

D

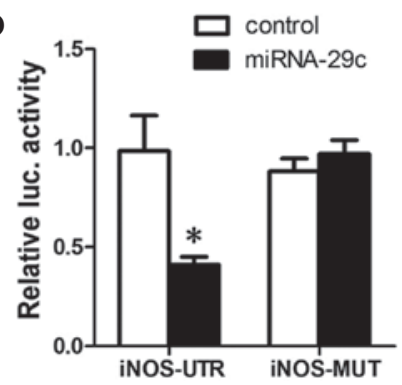

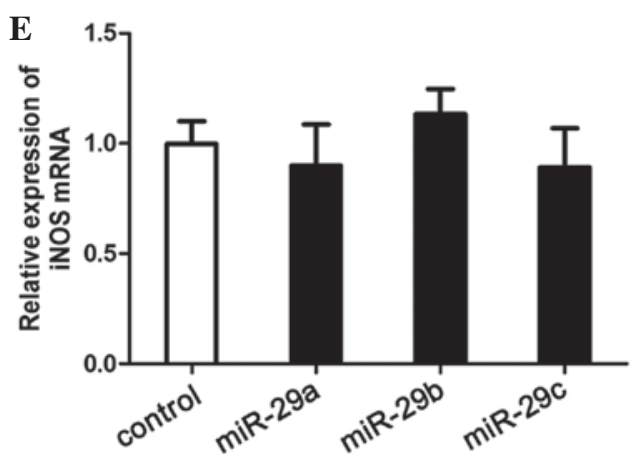
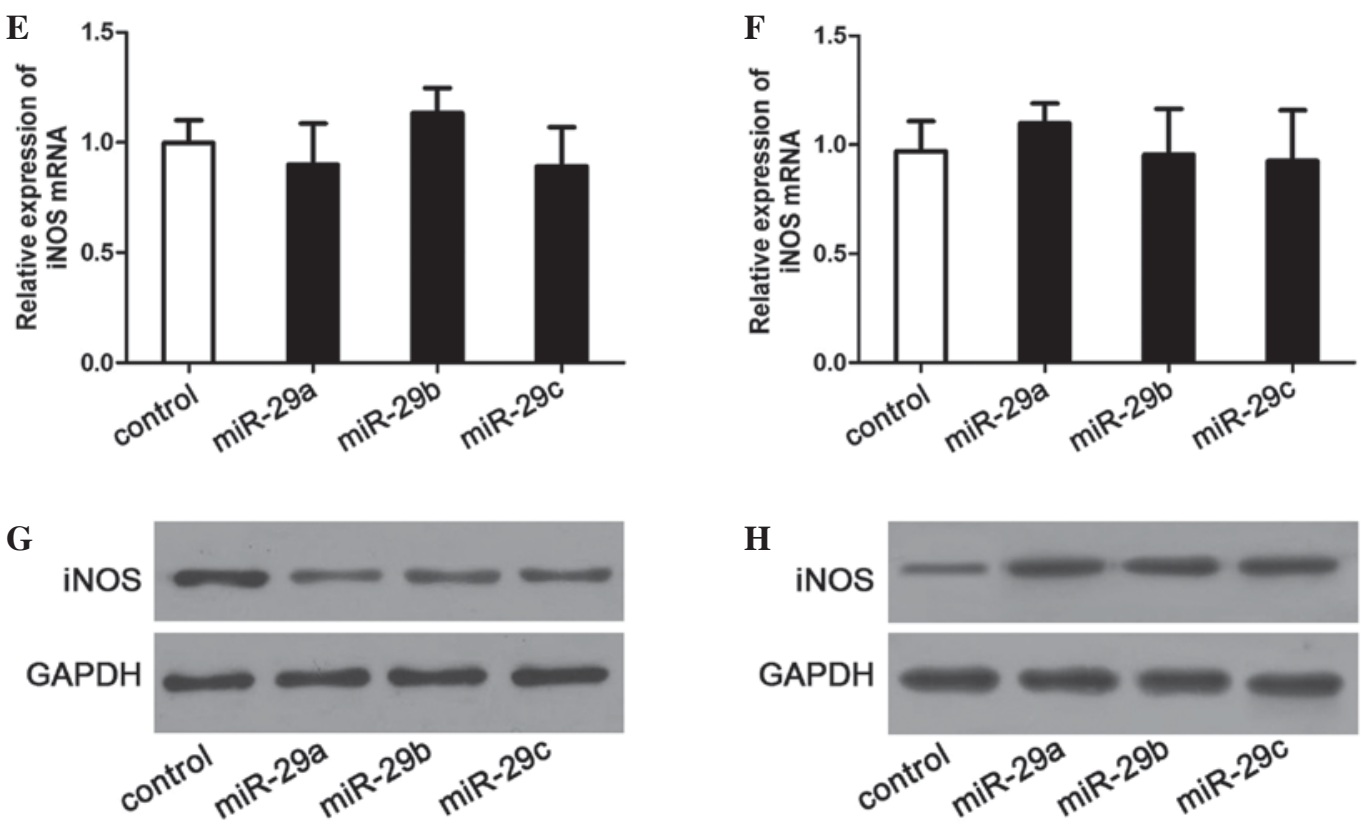

Figure 4. miR-29a/b/c targets iNOS. (A) Plasmids containing (a) wild-type or (b) mutant 3'UTR of iNOS sequences were constructed. (B) miR-29a, (C) miR-29b and (D) miR-29c significantly reduced the luciferase activity of the iNOS 3'-UTR plasmid, whereas the activity of the plasmid containing the mutant sequence was not significantly affected. Neither (E) miR-29a/b/c mimics nor (F) miR-29a/b/c inhibitors significantly affected the iNOS mRNA levels. Data are presented as the mean + standard deviation $\left(n=3 ;{ }^{*} \mathrm{P}<0.001\right.$ vs. control group). Western blotting showed that $(\mathrm{G})$ miR-29a/b/c mimics downregulated the expression levels of the iNOS protein, whereas $(\mathrm{H}) \mathrm{miR}-29 \mathrm{a} / \mathrm{b} / \mathrm{c}$ inhibitors increased the iNOS protein level. miR-29, microRNA-29; iNOS, inducible nitric oxide synthase; UTR, untranslated region; MUT, mutant.

of $\mathrm{miR}-29 \mathrm{a} / \mathrm{b} / \mathrm{c}$ was therefore explored. Potential miR-29a/b/c recognition sites in the iNOS promoter were identified using the TargetScan website (www.targetscan.org), and plasmids containing a matching site or mutant site were subsequently constructed. The sequences of these sites are listed in Fig. 4A. To establish a direct molecular link between miR-29a/b/c and iNOS, a luciferase reporter assay was performed. The data indicated that, compared to control plasmids, transfection with miR-29a/b/c mimics significantly decreased the luciferase activities of plasmids containing the wild-type iNOS 3'-UTR
$(\mathrm{P}<0.001)$, whereas the activities of plasmids containing the mutant iNOS 3'-UTR sequence were not markedly affected [relative fluorescence intensities: miR-29a, 0.99 \pm 0.08 $(\mathrm{P}=0.1349)$; miR-29b, $0.99 \pm 0.09$ ( $\mathrm{P}=0.1607)$; miR-29c, $0.97 \pm 0.07$ ( $\mathrm{P}=0.1824)$ ] (Fig. 4B-D).

To examine the effect of miR-29a/b/c on endogenous iNOS mRNA expression, RT-qPCR was used to detect changes in the mRNA expression levels of iNOS in $\mathrm{miR}-29 \mathrm{a} / \mathrm{b} / \mathrm{c}$ mimic-transfected or inhibitor-transfected cells. The results revealed no significant differences between the 
miRNA-mimic-transfected groups and the control groups $(\mathrm{P}=0.4662$ for miR-29a; $\mathrm{P}=0.1999$ for miR-29b; and $\mathrm{P}=0.4183$ for miR-29c; Fig. 4E). In addition, no significant differences were observed when comparing the miR-inhibitor-transfected groups and the control groups $(\mathrm{P}=0.2472$ for $\mathrm{miR}-29 \mathrm{a}$; $\mathrm{P}=0.9159$ for miR-29b; $\mathrm{P}=0.7879$ for miR-29c; Fig. $4 \mathrm{~F})$. Finally, the effects of miR-29a/b/c on the iNOS protein levels were examined. As shown in Fig. $4 \mathrm{G}$ and $\mathrm{H}$, transfection with the $\mathrm{miR}-29 \mathrm{a} / \mathrm{b} / \mathrm{c}$ mimics downregulated iNOS protein expression levels, whereas transfection with the miR-29a/b/c inhibitors increased the iNOS protein levels. These results indicate that miR-29a, b and c target and downregulate the iNOS protein, but not iNOS mRNA.

\section{Discussion}

The present study demonstrated that RIPC has a protective effect on NAFLD liver IR injury. In addition, the data revealed that RIPC reduced the $\mathrm{miR}-29 \mathrm{a} / \mathrm{b} / \mathrm{c}$ expression levels in skeletal muscle and increased the protein levels of iNOS; these results suggested that miR-29a/b/c targets iNOS, which plays an important role in the protective effect of RIPC during NAFLD liver IR injury.

In recent decades, IR injury has become a topic of particular interest in the context of liver surgery and research. Studies have predominantly focused on RIPC, which is a strategy for harnessing the body's endogenous protective capabilities against the injury that is incurred during IR (5-17). NO is an essential mediator of the protection that is afforded by hind-limb RIPC against liver IR injury (25). The mechanisms underlying this protection involve the preservation of the sinusoidal structure and the maintenance of blood flow through the hepatic microcirculation (25). The NO level is predominantly influenced by iNOS (27). NO is a volatile gas, and it is possible to monitor its release by measuring the mRNA and protein levels of iNOS (28). High expression of iNOS plays a protective role in IR (29).

In the current study, the liver condition of the NAFLD rats of the RIPC+IR group was better than that of NAFLD rats of the IR group. In addition, the liver cell apoptosis ratio was significantly lower in the RIPC+IR group compared with the IR group, supporting the concept of a protective role of RIPC in NAFLD liver IR injury. The iNOS protein level was significantly increased in the RIPC and RIPC+IR groups compared with the control group, suggesting that NO is an important mediator of the protective effect of RIPC during NAFLD liver IR injury.

RIPC can increase the NO level (25). However, the specific mechanism underlying this increase is not clear. In the present study, miR-29a/b/c levels were determined to be decreased in skeletal muscle cells of animals subjected to RIPC. miR-29 may serve tumor-suppressive and tumorigenic roles in cancer; thus, the expression of miR-29 may depend on the tissue and cellular context (30). It has been reported that RIPC protects the ischemic tissue from IR injury via a reactive oxygen species (ROS)-dependent pathway $(11,31,32)$, and that oxidative stress alters miRNA expression $(33,34)$. In the present study, oxidative stress or ROS were not measured. However, combining our data with that of previous reports, we speculate that the decrease in the $\mathrm{miR}-29 \mathrm{a} / \mathrm{b} / \mathrm{c}$ levels may be a result of oxidative stress due to the generation of ROS during RIPC.

To investigate the association between the increased iNOS and decreased miR-29a/b/c levels, a dual luciferase reporter assay was performed, which indicated that miR-29a/b/c directly targets iNOS. Notably, in a previous study, a significant increase in miR-29b and decrease in miR-29c expression was observed in the spleen following Cryptosporidium parvum-induced inflammation in C57BL mice, as compared with the controls. However, no significant change was observed in the expression of miR-29b/c in iNOS-knockout mice, suggesting that iNOS is required for the Cryptosporidium parvum-induced increase in miR-29b and decrease in miR-29c (35); this indicates that the regulatory mechanism between iNOS and miR-29a/b/c is complex and may involve a feedback loop. Further studies are therefore required to investigate the association between iNOS and $\mathrm{miR}-29 \mathrm{a} / \mathrm{b} / \mathrm{c}$.

In conclusion, the current study demonstrated that RIPC has a protective effect against NAFLD liver IR injury. This effect may occur due to a reduction of miR-29a/b/c levels in skeletal muscle, leading to increases in iNOS and, subsequently, NO. RIPC should be investigated further as a potential alternative protective strategy during NAFLD liver IR injury.

\section{Acknowledgements}

This work was supported by grants from the National Natural Science Foundation of China (no. 81502002), Applied Basic Research Project of Changzhou (no. CJ20140023) and Changzhou High-Level Medical Talents Training Project (no. 2016CZBJ044).

\section{References}

1. Lomonaco R, Sunny NE, Bril F and Cusi K: Nonalcoholic fatty liver disease: Current issues and novel treatment approaches. Drugs 73: 1-14, 2013.

2. de Meijer VE, Kalish BT, Puder M and Ijzermans JN: Systematic review and meta-analysis of steatosis as a risk factor in major hepatic resection. Br J Surg 97: 1331-1339, 2010.

3. Marsman HA, Heger M, Kloek JJ, Nienhuis SL, ten Kate FJ and van Gulik TM: Omega-3 fatty acids reduce hepatic steatosis and consequently attenuate ischemia-reperfusion injury following partial hepatectomy in rats. Dig Liver Dis 43: 984-990, 2011.

4. Song X, Xu H, Feng Y, Li X, Lin M and Cao L: Protective effect of grape seed proanthocyanidins against liver ischemic reperfusion injury: Particularly in diet-induced obese mice. Int J Biol Sci 8: 1345-1362, 2012.

5. Gassanov N, Nia AM, Caglayan E and Er F: Remote ischemic preconditioning and renoprotection: From myth to a novel therapeutic option. J Am Soc Nephrol 25: 216-224, 2014.

6. Manchurov V, Ryazankina N, Khmara T, Skrypnik D, Reztsov R, Vasilieva E and Shpektor A: Remote ischemic preconditioning and endothelial function in patients with acute myocardial infarction and primary PCI. Am J Med 127: 670-673, 2014.

7. Schmidt MR, Støttrup NB, Michelsen MM, Contractor H, Sørensen KE, Kharbanda RK, Redington AN and Bøtker HE: Remote ischemic preconditioning impairs ventricular function and increases infarct size after prolonged ischemia in the isolated neonatal rabbit heart. J Thorac Cardiovasc Surg 147: 1049-1055, 2014.

8. Crimi G, Pica S, Raineri C, Bramucci E, De Ferrari GM, Klersy C, Ferlini M, Marinoni B, Repetto A, Romeo M, et al: Remote ischemic post-conditioning of the lower limb during primary percutaneous coronary intervention safely reduces enzymatic infarct size in anterior myocardial infarction: A randomized controlled trial. JACC Cardiovasc Interv 6: 1055-1063, 2013. 
9. Cai ZP, Parajuli N, Zheng X and Becker L: Remote ischemic preconditioning confers late protection against myocardial ischemia-reperfusion injury in mice by upregulating interleukin-10. Basic Res Cardiol 107: 277, 2012.

10. Wever KE, Warlé MC, Wagener FA, van der Hoorn JW, Masereeuw R, van der Vliet JA and Rongen GA: Remote ischaemic preconditioning by brief hind limb ischaemia protects against renal ischaemia-reperfusion injury: The role of adenosine. Nephrol Dial Transplant 26: 3108-3117, 2011.

11. Dong HL, Zhang Y, Su BX, Zhu ZH, Gu QH, Sang HF and Xiong L: Limb remote ischemic preconditioning protects the spinal cord from ischemia-reperfusion injury: A newly identified nonneuronal but reactive oxygen species-dependent pathway. Anesthesiology 112: 881-891, 2010.

12. Zhou W, Zeng D, Chen R, Liu J, Yang G, Liu P and Zhou X: Limb ischemic preconditioning reduces heart and lung injury after an open heart operation in infants. Pediatr Cardiol 31: 22-29, 2010.

13. Rentoukas I, Giannopoulos G, Kaoukis A, Kossyvakis C, Raisakis K, Driva M, Panagopoulou V, Tsarouchas K, Vavetsi S, Pyrgakis V and Deftereos S: Cardioprotective role of remote ischemic periconditioning in primary percutaneous coronary intervention: Enhancement by opioid action. JACC Cardiovase Interv 3: 49-55, 2010.

14. Kanoria S, Jalan R, Davies NA, Seifalian AM, Williams R and Davidson BR: Remote ischaemic preconditioning of the hind limb reduces experimental liver warm ischaemia-reperfusion injury. Br J Surg 93: 762-768, 2006.

15. Tapuria N, Junnarkar SP, Dutt N, Abu-Amara M, Fuller B, Seifalian AM and Davidson BR: Effect of remote ischemic preconditioning on hepatic microcirculation and function in a rat model of hepatic ischemia reperfusion injury. HPB (Oxford) 11: 108-117, 2009.

16. Wang M, Shen J, Feng B, Gui L, Chen Q, Zhang B, Tang J and Li X: Remote ischemic preconditioning promotes early liver cell proliferation in a rat model of small-for-size liver transplantation. J Surg Res 179: e245-e253, 2013.

17. Abu-Amara M, Yang SY, Quaglia A, Rowley P, Tapuria N, Seifalian AM, Fuller BJ and Davidson BR: Effect of remote ischemic preconditioning on liver ischemia/reperfusion injury using a new mouse model. Liver Transpl 17: 70-82, 2011.

18. Jensen RV, Zachara NE, Nielsen PH, Kimose HH, Kristiansen SB and $\mathrm{B} \emptyset$ tker HE: Impact of O-GlcNAc on cardioprotection by remote ischaemic preconditioning in non-diabetic and diabetic patients. Cardiovasc Res 97: 369-378, 2013.

19. Contractor H, Støttrup NB, Cunnington C, Manlhiot C, Diesch J, Ormerod JO, Jensen R, Bøtker HE, Redington A, Schmidt MR, et al : Aldehyde dehydrogenase-2 inhibition blocks remote preconditioning in experimental and human models. Basic Res Cardiol 108: 343, 2013.

20. Abu-Amara M, Yang SY, Quaglia A, Rowley P, Tapuria N, Fuller B, Davidson B and Seifalian A: The hepatic soluble guanylyl cyclase-cyclic guanosine monophosphate pathway mediates the protection of remote ischemic preconditioning on the microcirculation in liver ischemia-reperfusion injury. Transplantation 93: 880-886, 2012.
21. Zitta K, Meybohm P, Bein B, Heinrich C, Renner J, Cremer J, Steinfath M, Scholz J and Albrecht M: Serum from patients undergoing remote ischemic preconditioning protects cultured human intestinal cells from hypoxia-induced damage: Involvement of matrixmetalloproteinase-2 and -9. Mol Med 18: 29-37, 2012

22. Li J, Xuan W, Yan R, Tropak MB, Jean-St-Michel E, Liang W, Gladstone R, Backx PH, Kharbanda RK and Redington AN: Remote preconditioning provides potent cardioprotection via PI3K/Akt activation and is associated with nuclear accumulation of $\beta$-catenin. Clin Sci (Lond) 120: 451-462, 2011.

23. Kanoria S, Glantzounis G, Quaglia A, Dinesh S, Fusai G, Davidson BR and Seifalian AM: Remote preconditioning improves hepatic oxygenation after ischaemia reperfusion injury. Transpl Int 25: 783-791, 2012.

24. Tapuria N, Junnarkar S, Abu-Amara M, Fuller B, Seifalian AM and Davidson BR: Modulation of microcirculatory changes in the late phase of hepatic ischaemia-reperfusion injury by remote ischaemic preconditioning. HPB (Oxford) 14: 87-97, 2012

25. Abu-Amara M, Yang SY, Quaglia A, Rowley P, de Mel A, Tapuria N, Seifalian A, Davidson B and Fuller B: Nitric oxide is an essential mediator of the protective effects of remote ischaemic preconditioning in a mouse model of liver ischaemia/reperfusion injury. Clin Sci (Lond) 121: 257-266, 2011.

26. Livak KJ and Schmittgen TD: Analysis of relative gene expression data using real-time quantitative PCR and the 2(-Delta Delta C(T)) Method. Methods 25: 402-408, 2001.

27. Michel T and Feron O: Nitric oxide synthases: which, where, how, and why? J Clin Invest 100: 2146-2152, 1997.

28. Alderton WK, Cooper CE and Knowles RG: Nitric oxide synthases: structure, function and inhibition. Biochem J 357: 593-615, 2001.

29. Phillips L, Toledo AH, Lopez-Neblina F, Anaya-Prado R and Toledo-Pereyra LH: Nitric oxide mechanism of protection in ischemia and reperfusion injury. J Invest Surg 22: 46-55, 2009.

30. Jiang H, Zhang G, Wu JH and Jiang CP: Diverse roles of miR-29 in cancer (review). Oncol Rep 31: 1509-1516, 2014.

31. Galagudza MM, Sonin DL, Vlasov TD, Kurapeev DI and Shlyakhto EV: Remote vs. local ischaemic preconditioning in the rat heart: infarct limitation, suppression of ischaemic arrhythmia and the role of reactive oxygen species. Int J Exp Pathol 97: 66-74, 2016.

32. Totzeck M, Hendgen-Cotta U, Rassaf T. Concepts of hypoxic NO signaling in remote ischemic preconditioning. World J Cardiol 7: 645-651, 2015

33. Fatemi N, Sanati MH, Shamsara M, Moayer F, Zavarehei MJ, Pouya A, Sayyahpour F, Ayat H and Gourabi H: TBHP-induced oxidative stress alters microRNAs expression in mouse testis. J Assist Reprod Genet 31: 1287-1293, 2014.

34. Wang Z, Liu Y, Han N, Chen X, Yu W, Zhang W and Zou F: Profiles of oxidative stress-related microRNA and mRNA expression in auditory cells. Brain Res 1346: 14-25, 2010.

35. Mathé E, Nguyen GH, Funamizu N, He P, Moake M, Croce CM and Hussain SP: Inflammation regulates microRNA expression in cooperation with $\mathrm{p} 53$ and nitric oxide. Int J Cancer 131: 760-765, 2012. 